\title{
DIAGNOSIS OF PERIODONTOCLASIA.
}

\author{
By John Oppie McCall, D. D.S., Buffalo, N. Y.
}

(Read before the National Dental Association at Its Twenty-third Annual Session, New Orleans, La., October 20-24, 1919.)

$\mathrm{D}$ IAGNOSIS is the foundation of treatment. It is the recognition of the fact that a disease process exists, and it designates the nature of the process. But diagnosis implies more than the recognition and delimitation of the pathological condition; it implies as well a determination of the etiological elements which are involved. It is to the physician. what the chart and compass are to the mariner. For if the mariner sets out without the necessary instruments of navigation, he seldom, if ever, reaches his port of destination.

Applied to the treatment of human disease, the methods of the incompetent mariner become nothing more nor less than mal-practice. Recognition of periodontal disease is so simple, that when present, it is obvious even to the lay observer. Unfortunately the dentist frequently is oblivious to its existence until the anxious patient intrudes the evidence of its presence upon his consciousness. The reason for this apathy lies perhaps in a series of unsuccessful attempts to control these disorders. It was said of Abraham Lincoln that he avoided the dance, for the reason doubtless that he sensed his deficiency in this art. So too, the dentist.

But the dentist while capable of discovering early signs of oral disease, has been lost in the maze of conflicting opinions regarding the nature of periodontoclasia, its etiology and its treatment. Moreover the text books of the past in dealing with this subject, under such titles as "pyorrhea alveolaria," "interstitial gingivitis," "phagedenic pericementitis," "suppurative pericementitis," "peridentitis," etc., have assumed a distinctly pessimistic attitude toward these disease processes.

In the light of this attitude as expressed by the recognized authorities, it is small wonder that the practitioner has developed a frame of mind where he not only hesitates to treat these conditions, but closes his eyes to their very existence.

Ability to diagnose periodontal disease has apparently been developed by a sort of inverse process. In the days of Riggs there was no diagnosis which was worthy of the name. With the gradual development in the art of periodontia, treatment has consistently led diagnosis. Advances in the methods of treatment have revealed the significance of pathological and etiological factors, thru which the development of diagnosis has followed. As a matter of fact, however, each advance in diagnosis has been brought about by the earnest study of previously inexplicable failures. Each difficulty when surmounted has proved proved a stepping stone to the solution of the next problem.

Strange to say the microscope which ushered in the era of modern pathology, as exemplified in the study of antiseptics and the bacterial anti-bodies of living tissues, diverted the forces of periodontic investigation into hopeful but un- 
productive channels. For while the phenomena of the breaking down and repair of periodontal tissue can only be fully understood thru the revelations of the microscope, it is nevertheless true that the bacteriological explanation of these phenomena constitutes but a chapter of the story. Students of inflamatory processes distinctly state that these changes may be brought about by mechanical, chemical or bacterial irritants. But enthusiasm over the discovery of bacterial elements in disease has blinded dental investigators to the coexistence of all three forms of irritation in diseases of the periodontium. The forces of repair, no matter how stimulated, can never successfully combat mechanical irritation thru a method of treatment aimed chiefly at bacterial irritants. Diagnosis therefore to be complete, must take into account all of the factors of disease and its causation, and assign to each its proper valuation in the group.

Diagnosis of advanced periodontoclasia is simple, because of the obvious breaking down of the tissues which support the teeth. Diagnosis at this period has little practical value of course, for the reason that when the condition has reached an advanced stage, repair is beyond the power of the organism.

The recognition of the early symptoms of periclasia is more difficult, yet the dentist must bend his energy toward its diagnosis at the earliest possible moment, and for two very cogent reasons. The first lies in the obvious advantage accruing to the patient from the favorable prognosis obtaining in practically every case which is discovered before any considerable dissolution of the supporting structures has taken place. The other has to do with the difficult technic and varied instrumentarium required for the root surgery of advanced cases. In other words, the average incipient case will be within the diagnostic powers of the competent general practitioner, and it will not demand a skill in treatment beyond his ability to acquire nor will it require a very considerable assortment of instruments. The field of the general practitioner is in the treatment of incipient cases: when he is dealing with lesions exhibiting a pocket depth of more than three millimeters, his chances of success begin to dwindle very rapidly. This assertion is made as a result of the experience gained in handling many such lesions, with a realization of the requirements of natural aptitude, exacting technic and assortment of scalers and other instruments, which these cases demand.

Diagnosis requires first a knowledge of the normal, the mouth in a state of health. Briefly, this means pink lustrous gingiva covering the cemento-enamel junction around the entire circumference of each tooth and filling the interdental spaces to the contact points of the teeth. An explorer slipped under the border of the marginal gingiva should penetrate no further than the junction of the enamel and cementum. The labial and lingual gingival tissue has an even contour as traced vertically from the buccal fold or from the roof of the mouth to the crest of the marginal gingiva, without any swelling or bellying out as it nears the crest. The marginal gingiva itself terminates in a thin knife-like edge. It should hardly be necessary to catalogue these points in detail, yet few adult mouths exhibit such a state of health as is here pictured. Thus we grow so accustomed to varying degrees of departure from the normal, as to consider these aberrations normal, until they become so obviously pathological as to challenge our attention.

To discover the presence of disease conditions of the gingival tissue then, is simple to the point of absurdity. It requires only a careful ocular examination supplemented by exploration under the gingival margin with a slender blunt 
probe. Differential diagnosis of the types of periodontoclasia and the determination of their etiology is not so simple yet even here careful study will enable the conscientious dentist to correctly diagnose most of the cases under his observation. Given a knowledge of the types of periclasia and their etiology the next requirement in diagnosis is optimism. The dentist when examining for the presence of gingival disease must not dread to find it, or he will inevitably blind himself to the first signs, and thus endanger the health of periodontal tissues which he himself most frequently may restore to a normal condition. He must approach his patient with the knowledge that periodontal disease can be cured, and can be prevented.

There is no one disease of the investing tissues of the teeth, which exhibits all the characteristics observed in the pathological processes which attack these tissues. If we are to say that there is one disease and that it is characterized by the presence of pus we are at once confronted by the fact that periodontal tissue may be broken down without the occurrence of this symptom. The same holds true for recession of the marginal gingiva, tooth mobility, visible inflammation, or in fact any other single manifestation which may be observed in cases of periclasia. Efforts to justify the absence of one or more supposedly cardinal symptoms from the typical disease have invariably failed, while but little better success has attended the effort to subdivide or classify what have been acknowledged to be two or more fairly distinct pathological processes. The stumbling block in the way of classification has been the almost invariable blending and intermingling of types, so that analysis of a case into its component disease types is extremely difficult. Another source of error is the temptation to classify by symptoms. The formation of pus, for instance, while symptomatic of certain types of disease is not to be taken as the distinguishing feature of those types; rather should we recognize as the distinguishing feature the tissue changes which make possible an infection by pyogenic bacteria. Another source of error is introduced when a classification is proposed which is based upon coincidental, but not necessarily fundamental phenomena. As an instance of this may be cited proposed classifications based upon the presence in the system of diseases such as diabetes, nephritis, senility, etc. The simplest method of classification is one based upon morphology and visible inflammation. Proceeding upon this plan we find that there are disease processes attacking primarily the gingival tissue, the pericementum or alveolar bone: these diseases may or may not cause visible inflammation. Thus it will be possible to have a destructive process attacking the marginal gingiva. If this is accompanied by redness, which is the only constant symptom found in inflammations of this tissue, we call this type gingivitis. If the symptom of redness is more or less lacking, we may find that the cardinal symptom is a dimunition in bulk of the gingival margin. This type is called recession. If the pericementum is broken down, whether accompanied by other tissue changes or not, we have a type which may properly be called pericementoclasia. The fact that destruction of bone is necessarily coincidental with this process, does not alter the fact the pericementum is the tissue principally invelved.

A breaking down of the alveolar process may take place without severance of the penetrating fibres of the pericementum. This type is called alveoclasia.

Inflammations of the periodontal tissues occur sometimes without a previous solution of continuity of these tissues. While these inflammations do not always proceed to the formation of a typical 
abscess we nevertheless are impelled to classify them as periodontal abscesses, for the reason that the essential nature of this disease type is the same whether the infecting bacteria cause the generation of pus or lead to the formation of granulomata, etc.

With the exception of recession the types mentioned seldom occur pure. They may be observed mingled in all possible commutations, and with the typal lines so indistinct that each case seems to present a somewhat different picture from the rest. Nevertheless by keeping in mind the anatomy of the parts and then examining the case to determine the tissues affected, together with the presence or absence of inflammatory symptoms, it is possible to differentiate and analyze each case into its basic and component types. The case will naturally be known by the name of the predominating type. For example: we may find a tooth whose periodontium exhibits a recession and also an inflammation of the marginal gingiva, together with the breaking down of the pericementum and of the alveolar bone with pus formation. The most prominent feature of this disease complex is suppuration and the severance of the penetrating fibres of the pericementum; because of this fact, we call this condition suppurative pericementoclasia, for which we also use the synonymous term suppurative periodontoclasia. The term pyorrhea alveolaris may also be used to designate this condition, altho it is in a strict sense incorrect, since study of the pathology of this disease type indicates that the pus originates in the gingival tissue overlying the denuded cementum and not in the alveolar bone. For these reasons we propose a classification based on the outstanding features of the several types, features by which they may usually be readily distinguished and differentiated. It will of course frequently occur that the characteristics of one type blend imper- ceptibly into another, making differentiation difficult and occasionally impossible. However, under these circumstances it is always possible to distinguish the most prominent feature of the case and designate the case by the name of the corresponding type.

It should also be noted that each of these types permits of subdivisions which are still further descriptive. They are usually based on etiological and other characteristics.

Study of the etiology of the various disease types reveals a fact which should be obvious, but which nevertheless has not been properly brought out, in the past, namely-that certain causative factors have a tendency to produce certain types of disease. Hence it will naturally follow that having determined the type or types present, their etiology is, to say the least, very strongly suggested.

In considering these types in detail we will enumerate for each its cardinal symptoms, its differentiating symptoms and its usual etiology. The length of this essay will not permit of a discussion of pathology treatment, or prognosis.

Recession. The cardinal symptoms are diminution in the bulk of the gingival tissue and the underlying crest of the alveolar process, with exposure of the cementum. In the pure form there is no redness, swelling, pain or pus formation: these symptoms differentiate it from other types of periclasia. Its etiology is largely traumatic. Traumatic occlusion is present in most cases, in combination with extra-oral trauma, particularly a vigorous but misdirected use of the tooth brush. Frail osseons support, insufficient blood supply and lack of normal function may be contributing causes. There is little, if any, invasion by bacteria. This is presumably due to the fact that the vigorous tooth-brush massage, which is nearly always found as a factor in the pure type, keeps the gingiva so 
toned up that they have a high immunity.

Chronic gingivitis is characterized chiefly by redness of the marginal and cemental gingival. There is usually some swelling of these tissues, also. But in the pure type both redness and swelling are confined the marginal and cemental gingiva, the alveolar gingiva remaining practically normal in color and contour. There may or may not be pain, but the marginal gingiva is more or less sensitive to touch. Pus if present is expressed in but very slight amount. The crest of the marginal gingiva is rounded, not having the thin edge characteristic of it in health, and it may be more or less everted from the tooth surface. Chronic gingivitis in pure form is differentiated from other types as follows: There is no exposure of cementum thru the receding of the gingival tissue: there is no pocket formation or severing of the penetrating fibres of the pericementum: pain is mild and subacute in character: radiographs show comparatively little absorption of the crest of the alveolar process or thickening of the pericementum: there is no mobility of the tooth. Chronic gingivitis is usually caused by the contact of some irritating substance with the marginal or cemental gingiva, as for instance unsanitary concretions on the tooth surface, a roughness of the tooth surface itself, or the impingement of rough or sharp edges of fillings, crown bands, etc. When traumatic occlusion is present, gingivitis will often be seen as a stage in the progression of events leading to suppurative pericementoclasia. When so occurring there is usually some tenderness of the tooth to percussion. Food impaction thru faulty contacts is a causative factor. Bacterial toxins localized by the various kinds of deposits play a definite part in the production of gingivitis, the bacteria being those of low virulence. Other forms of chemical irri- tation may occasionally be found, to. gether with faulty local circulation. These latter irritants, as also the products of disease conditions elsewhere in the body, are distinctly contributing and not inaugurating factors. Epithelial faults in the gingival crevice and habits such as mouth-breathing will also contribute to the production of gingivitis.

The diagnosis of chronic gingivitis, and its etiology is of great importance since it very commonly occurs as an introductory lesion preceding a deeper destruction of the periodontal tissues. We cannot take this as an invariable sequence, however, and allow a pink gingival tissue to lull our suspicions, since deep pocket formation may take place without any evidence of gingivitis becoming visible at any stage of its development.

Hypertrophic gingivitis is a form of chronic gingivitis. It presents the symptoms of the chronic type in addition to which there is visible hypertrophy of the tissue elements. It is caused chiefly by contact of a mechanical appliance with the marginal gingiva, but without pressure. A typical instance is a crown band extended under the marginal gingiva, which does not hug the periphery of the tooth closely, but which does not extend far enough to press against the fibres of attachment of the cemental gingiva. Being of polished metal, the band does not act to any extent as a carrier for bacterial colonies. Hence, in the presence of the high bacterial resistance usually found in these cases, there may be comparatively little redness of the hypertrophied tissue. Indolent gingivitis is another form of chronic gingivitis. It is characterized by the bright red color of the marginal gingiva, as contrasted with the purplish red of the usual chronic case. It is usually more or less painful. It is differentiated from the acute ulcerative gingivitis by the absence of greyish necrotic tissue overlying the 
reddened gingiva, by the mild character of the pain when present, and by the absence of the fetor of the breath characteristic, the latter condition. Its etiology is a bacterial attack following mechanical irritation such as traumatic occlusion, presence of deposits, edges of crowns, etc. In this type the tone of the tissues is below normal, hence the bacteria are not readily eliminated from the tissues, even after the relief of the primary mechanical irritant. These cases consequently show a persistent altho a modified redness for some time after treatment has been given. Systemic disorders may be a complicating factor.

The symptoms of acute ulcerative gingivitis or Vincent's gingivitis are: a grayish white ulcer on the crest of the marginal gingiva, either labial lingual or proximal, with a bright red line immediately under it: constant severe pain and tenderness to touch: characteristic fetor of the breath: hemorrhages at the slightest touch or even spontaneous; more or less fever and mental depression. The onset is sudden. There is often loss of appetite, insomnia, salivation, and the patient may be incapacitated for work. It is differentiated from other types by the foregoing symptoms, all of which are characteristic. There is no visible pus: the teeth are usually firm, and there are no pockets unless these phenomena have been brought about by previously existing periclasia. There is no recession unless it has preceded the attack. Acute ulcerative gingivitis is caused by an infection of Vincent's fusiform bacillus and spirochete. There is usually a previous preparation of the tissues by mechanical irritants, such as traumatic occlusion, presence of deposits, and edges of crowns and fillings in contact with the gingiva, impaction of food against the proximal gingiva or sometimes the labial or lingual gingiva. It is often found in the mouths of syphilitics, but the occurrence of Vincent's infection gives no grounds for suspecting specific disease. Cigarette smoking is a predisposing cause.

Alveoloclasia is a type of peridontoclasia which seldom occurs in the pure form as a pathological phenomenon, altho it is frequently produced temporarily in the performance of dental operations. Its symptoms are mobility of the tooth in its socket, and absorption of the inner wall of the alveolar bone or of varying portions of the alveolar wall en masse as revealed by the radiograph. Recession of the marginal gingiva is so constantly found as to require mention as a coincident phenomenon. It is differentiated from the other types by the normal color of the gingival tissue, and by integrity of pericementum. In other words there is no visible inflammation, nor is there any formation of a so-called' pocket. There is no pain or tenderness, nor is there any pus formation.

Alveoloclasia as a pathological process is caused in the majority of cases by traumatic occlusion. It is also a result of congenital non-disocclusion, being one of the phenomena of the resultant disuse atrophy. There is little or no bacterial invasion, this being due to high resistance in the tissues. Frail osseous support and lowered systemic condition may complicate cases in which it occurs with other types. It is produced artificially by the separation of teeth for filling operations and by the pressure of orthodontic appliances. When these forms of pressure are properly applied it does not become pathological and is of course a necessary antecedent to the desired movement of the teeth. Improper application of force or lack af proper post-operative retention, however, may readily lead to the establishment of a pathological condition.

Periodontal abscess is a term applied to any infection of the periodontal tissues, whose inflammatory products do not find an exit thru the gingival crevice. 
The form arising from infection of the contents of the pulp canal are outside of the scope of this paper. Parietal or extra-pulpal abscesses are of two kindspericemental and gingival. The symptoms of pericemental abscess are much the same as those of the acute apical alveolar abscess, namely: pain, tenderness of the tooth to percussion, redness and swelling of the alveolar gingiva, elongation of the tooth in its socket and finally pointing, and discharge of pus thru the alveolar gingiva, not at the gingival crevice. It is differentiated from apical alveolar abscess by occurring around the root of a tooth having a vital healthy pulp, or possibly around a nonvital but well filled tooth, the radiograph of which shows a definite point origin along the coronal half of the root. It is also differentiated by the prompt subsidence of symptoms and return to health without resort to pulp canal treatment. It is differentiated from gingival parietal abscess by occurring in a previously intact pericementum, in other words in a location where there is no so-called pocket. It is caused by a hematogenous infection in a portion of the pericementum which has been subjected to the irritation of an occlusal trauma.

Gingival parietal abscess is characterized by having its point of origin in the gingival tissue overlying a pocket or follicle, and is consequently a sequel to pericementoclasia. It presents symptoms of pain, redness and swelling of the alveolar gingiva, with pointing at a spot somewhat removed from the margin. It may occur around a vital or a non-vital tooth. It is differentiated from apical alveolar abscess and from pericemental abscess in that it is located in gingival tissue over a so-called pocket, and not in pericemental tissue. It is usually caused by a retention of the products of the previously existing infection, together with the bacterial proliferation within the tissue which such retention favors. This retention of the by-products of infection may be brought about in a mechanical way by the presence of excess filling material or the pressure of a band against the gingival tissue. Or it may occur as the result of a stasis due to the effect on the circulation of some systemic disorder, as for instance diabetes. But even in such cases there is always a preceding mechanical factor which has produced a congestion of the blood vessels.

Suppurative pericementoclasia is characterized by the formation of a so-called pocket thru the severance of the penetrating fibres of the pericementum without coincident loss of the overlying gingival tissue. This condition is readily discovered by exploration with a fine blunt probe. There is a corresponding loss of alveolar bone which is usually absorbed a little ahead of the severance of pericemental, fibres so that pocket or follicle formation seldom exposes the alveolar bone to the contents of the pocket. The marginal gingiva is slightly thickened and its crest presents a blunt edge as in gingivitis, but redness in the pure type is lacking. There is more or less pus formation proceeding from the inner gingival wall of the pocket, and exuding at the gingival margin. In the pure form it is differentiated from other types by lack of tooth mobility: the gingival tissue occupies its usual position over the tooth root in spite of the loss of connecting pericemental fibres: there is no redness of the marginal gingiva: there is no pain and the body temperature is normal. These constitute the symptoms of suppurative pericementoclasia. There may be coincident recession and gingivitis with or without pain, and there may be sufficient absorption of the inner wall of the alveolus to permit the tooth to move perceptibly in its socket, in other words alveoloclasia. Abscess formation may take place in the gingival tissue overlying the periclasial pocket. These things are coincidental with, but not a necessary 
part of pericementoclasia. When they do so occur they may be considered secondary to this type. Hence when a periclasial follicle or pocket has been formed by the destruction of the pericemental fibres, the case will be diagnosed as pericementoclosia even in the presence of other types of periodontoclasia.

Suppurative pericementoclasia has as primary causative factors, traumatic occlusion and food impaction where approximal contents are insufficient to protect the gingival tissue. This latter factor, however, is not so common a cause of this type of disease as has been held in the past, nor does it produce such deep lesions as that arch evildoer, traumatic occlusion. Secondary factors are the deposits of serumnal calculus which are formed as a result of the irritation and congestion produced by the primary factors. Invasion of the irritated tissues by pyogenic bacteria is responsible for the severence of pericemental fibres and the formation of pus. As this invasion is only possible when the tone of the tissues has been lowered by the primary irritation, bacteria are also rated as secondary factors. Other secondary factors are cell vitality and the various factors which affect it, organic chemical irritants, and rarely, inorganic irritants. Mechanical irritants acting on the marginal and cemental gingiva are not factors in the production of typical pericementoclasia, but if present will of course tend to produce modifications from the pure type according to the nature of irritant.
Results in the treatment of dental periclasia depend on three factors-correct diagnosis, correct treatment and correct prognosis. Treatment and prognosis cannot be considered without unduly lengthening this paper. But attention should be called to this fact, namely: that failure to obtain successful results in the handling of a case indicates a failure in one of these three departments. While this statement is so obvious as to verge on the inane, it nevertheless has a very real meaning to the practitioner. In the past our profession has looked at periclasia with a desperate but pessimistic eye, and whether success or failure attended our efforts, there seemed no explanation to offer. Our attitude was fatalistic to a degree.

But with an understanding of diagnosis and etiology, our attitude changes. We know that success in treatment is possible. Therefore if we fail in a case whose prognosis is favorable, we can soon determine whether our shortcomings were in the field of diagnosis or in treatment. Needless to say, when we find ourselves able to diagnose our cases correctly and determine definitely their etiology, we will bend ourselves with the energy and ability characteristic of our profession, to attaining that perfection in treatment by which we may give our patients the oral health they have a right to expect. 